\title{
All-Pairs Shortest Paths Algorithm for High-dimensional Sparse Graphs
}

\author{
Urakov A. R., Timeryaev T. V.* \\ General Scientific Faculty, Ufa State Aviation Technical University, Ufa, 450077, Bashkortostan, Russia \\ *Corresponding Author: timeryaev@yandex.ru
}

Copyright (C2013 Horizon Research Publishing All rights reserved.

\begin{abstract}
Here the All-pairs shortest path problem on weighted undirected sparse graphs is being considered. For the problem considered, we propose "disassembly and assembly of a graph" algorithm which uses a solution of the problem on a small-dimensional graph to obtain the solution for the given graph. The proposed algorithm has been compared to one of the fastest classic algorithms on data from an open public source.
\end{abstract}

Keywords APSP, graph disassembly, graph assembly, graph contraction, sparse graphs

\section{Introduction}

The APSP (all-pairs shortest path problem) is one of the most popular tasks in graph theory because the shortest paths between all pairs of vertices are used for solving many problems involving discrete optimization (TSP, theory of transportation task etc). Moreover, the task itself is of great interest in research.

Recently this problem has gained new interest due to a growing number of highly detailed graphs that are generated automatically and describe structures from the real world. Such graphs have about $10^{6}$ or more vertices and this number will inevitably increase. So the acceleration of APSP solving for high-dimensional graphs is becoming highly important.

Because of its popularity, there are a lot of APSP solution algorithms but there isn't any method to obtain the solution as fast for different kinds of input data. That's why APSP solution algorithms can be classified according to the type of graph as follows: directed [3], complete [5], weighted [4], unweighted [1] and sparse [7].

Here we present an algorithm for solving the APSP for weighted, undirected and high-dimensional sparse graphs with non-negative weights.

This paper is organized as follows. In section 2, we introduce notation and the problem definition, in section 3 , we describe the algorithm and in section 4 we show the results in comparison with one of the most renowned APSP algorithms.

\section{Notation and problem defini- tion}

\subsection{Terms and definitions}

Here, we consider a connected, undirected and sparse graph $G=(V, E, w)$, where each edge $e\left(v_{i}, v_{j}\right)$ has a non-negative weight $w(i, j)$. The given graph $G$ is considered to be simple (has no loops or multiple edges).

Denote by $|V|=n$ the order of a graph or cardinality of vertices set. Denote by $|E|=m$ the size of a graph or cardinality of edges set.

Denote by $w(i, j)$ the weight of the edge between vertices $v_{i}$ and $v_{j}(w(i, j)=\infty$, for non-connected vertices). $A$ degree $d\left(v_{i}\right)$ of vertex $v_{i}$ is the number of edges incidental to $v_{i}$. A graph is called sparse if $m \ll n^{2}$.

$A$ path is an alternating sequence of vertices and edges $v_{0}, e_{1}, v_{1}, \ldots, v_{k-1}, e_{k}, v_{k}$, beginning and ending with a vertex. In that sequence, each vertex is incidental to both the edge that precedes it and the edge that follows it. A length of a path is the sum of the weights of its edges. A distance $m(i, j)$ between $v_{i}$ and $v_{j}$ is the length of the shortest path $p_{i j}^{s}=p^{s}\left(v_{i}, v_{j}\right)$ between these vertices. A distance matrix is a matrix in which each element at the intersection of $i$ th row and $j$ th column contains the length of the shortest path between $v_{i}$ and $v_{j}$. A graph is said to be connected if every pair of vertices in the graph is connected by some path, i.e. $m_{i j}<\infty, \forall i, j$.

Between any pair of vertices there can be more than one shortest path. We do not consider it as an essential issue in this paper, so the references to the shortest path can mean any of them.

A matrix is called a precedence matrix if each element $p_{i j}$ of the matrix corresponds to the vertex that precedes vertex $v_{j}$ in the path from $v_{i}$ to $v_{j}$. Therefore the elements of $P$ can be determined by

$$
p_{i j}= \begin{cases}v_{k}, & \exists v_{k}: p_{i j}^{s}=\ldots v_{k}, e\left(v_{k}, v_{j}\right), v_{j} \\ \infty, & \text { else }\end{cases}
$$

Using $P$ the shortest path $p_{i j}^{s}$ from $v_{i}$ to $v_{j}$ in a connected graph can be obtained by the recursive formula:

$$
p_{i j}^{s}= \begin{cases}p^{s}\left(v_{i}, p_{i j}\right), e\left(p_{i j}, v_{j}\right), v_{j}, & p_{i j} \neq v_{i} \\ v_{i}, e\left(v_{i}, v_{j}\right), v_{j}, & p_{i j}=v_{i}\end{cases}
$$


Now, we shall give the following supplementary definitions. Let us call a graph sequence $S=$ $\left\{G_{1}, G_{2}, \ldots, G_{r}\right\}$ shrinking graph $G_{0}=\left(V_{0}, E_{0}, w_{0}\right)$, where $G_{p}=\left(V_{p}, E_{p}, w_{p}\right), V_{p}=\left\{v_{1}^{p}, v_{2}^{p}, \ldots, v_{n(p)}^{p}\right\}$, $E_{p}=\left\{e_{1}^{p}, e_{2}^{p}, \ldots, e_{m(p)}^{p}\right\}: e_{i}^{p}=e^{p}\left(v_{j}^{p}, v_{k}^{p}\right) \subseteq V_{p} \times V_{p}$ and $w_{p}: E_{p} \rightarrow[0, \infty)$.

Every next graph $G_{p+1}$ of the sequence is obtained from the previous $G_{p}$ by the removing the $k$ vertices and the edges incidental to them, plus the addition of new edges and by recalculating the weights of the edges adjacent to the deleted ones.

For these graphs, we get $\left|V_{p}\right|>\left|V_{p+1}\right|, \forall p=\overline{0, r-1}$. Denote by $v_{i}^{p+1}$ a vertex of $G_{p+1}$ corresponding to vertex $v_{i}^{p}$ of $G_{p}$. Denote by $e^{p+1}\left(v_{j}^{p+1}, v_{k}^{p+1}\right)$ an edge of $G_{p+1}$ corresponding to the edge $e^{p}\left(v_{j}^{p}, v_{k}^{p}\right)$ of $G_{p}$.

Denote by $G_{p+1}=R_{p}\left(v_{1}^{p}, v_{2}^{p}, \ldots, v_{k}^{p}\right)$ the graph obtained from $G_{p}$ by removing the vertices $v_{1}^{p}, v_{2}^{p}, \ldots, v_{k}^{p}$ and the edges incidental to them. For this graph we get $w_{p+1}(i, j)=w_{p}(i, j), \forall i, j: v_{i}^{p+1}, v_{j}^{p+1} \in V_{p+1}$.

Denote by $m^{p}(i, j)$ the distance between $v_{i}^{p}$ and $v_{j}^{p}$ in $G_{p}$. By $M_{p}=\left(m_{i j}^{p}\right)$ denote the distance matrix of $G_{p}$. Also denote by $v_{i_{l}}^{p} l$ th adjacent to $v_{i}^{p}$ vertex and by $A_{i}^{p}$ the set of all adjacent to $v_{i}^{p}$ vertices in graph $G_{p}$.

\subsection{Problem definition}

Given a connected, undirected, simple, weighted and sparse graph $G=(V, E, w)$, where each edge has a nonnegative weight $w: E \rightarrow[0, \infty)$. Find the shortest paths between every pair of vertices of the graph, i.e. find the distance matrix $M$ and the precedence matrix $P$ of the graph.

\section{Algorithm of the solution}

\subsection{Main idea}

The main idea of the introduced algorithm is to reduce the problem on a large graph to the problem on a smaller graph. The algorithm can be partitioned into 3 stages.

1. Compression. A large initial graph is replaced by a small graph.

2. Microsolution. The APSP for the small graph is solved by using any known method.

3. Restoring. The APSP solution for the small graph is projected onto the initial graph.

While using this method we must satisfy the following conditions: a) validity "- the compression must keep information about the shortest paths of the initial graph; b) efficiency "- the introduced method must be quicker than all others.

The algorithm in which similar ideas were used are considered in [6]. Here we introduce an algorithm of a graph disassembly/assembly for large sparse graphs. At the disassembly stage, we consistently remove vertices, and then solve the APSP for the resulting small graph. At the assembly stage the initial graph is restored with the calculation of distances and paths.

\subsection{Disassembly}

The disassembly stage consists of consistent approximation of the initial graph $G_{0}=\left(V_{0}, E_{0}, w_{0}\right)$ by the graphs of a shrinking sequence $S=\left\{G_{1}, G_{2}, \ldots, G_{r}\right\}$. Here we consider a particular case in which every next graph $G_{p+1}$ of the sequence $S$ is obtained by removing only one vertex from $G_{p}$.

Suppose that vertex $v_{i}^{p}$ is to be removed. Let the degree of $v_{i}^{p}$ be equal to $k$. If any shortest path contains $v_{i}^{p}$ (except shortest path straight to or from $v_{i}^{p}$ ) then this path contains subpath $v_{i_{j}}^{p}, e^{p}\left(v_{i_{j}}^{p}, v_{i}^{p}\right), v_{i}^{p}, e^{p}\left(v_{i}^{p}, v_{i_{l}}^{p}\right), v_{i_{l}}^{p}$ : $j, l \in\{1,2, \ldots, k\}$. Therefore to remove vertex $v_{i}^{p}$ properly, we need to preserve the shortest paths only between vertices adjacent to $v_{i}^{p}$.

By

$$
w_{p}^{\operatorname{mv}(1,2, \ldots, h)}\left(i_{j}, i_{l}\right)
$$

$\min _{g=1,2, \ldots, h}\left(w_{p}\left(i_{j}, g\right)+w_{p}\left(g, i_{l}\right)\right)$ denote the minimum sum of the weights of two edges which connect vertices $v_{i_{j}}^{p}, v_{i_{l}}^{p}$ and are incidental to a common vertex that belongs to the set $v_{1}^{p}, v_{2}^{p}, \ldots, v_{h}^{p}$ of $G_{p}$. To preserve distances it is sufficient to have

$$
w_{p+1}\left(i_{j}, i_{l}\right)=\left\{\begin{array}{l}
\min \left(w_{p}^{\operatorname{mv}(i)}\left(i_{j}, i_{l}\right), w_{p}\left(i_{j}, i_{l}\right)\right), \\
\text { if } w_{p}^{\operatorname{mv}(i)}\left(i_{j}, i_{l}\right)<w_{p}^{\operatorname{mv}(h \neq i)}\left(i_{j}, i_{l}\right) \\
w_{p}\left(i_{j}, i_{l}\right), \text { else }
\end{array}\right.
$$

for any pair $\left(v_{i_{j}}^{p}, v_{i_{l}}^{p}\right)$ in $G_{p+1}$.

At the beginning of the algorithm any element of $P^{\prime}$ is equal to infinity $p_{i j}^{\prime}=\infty, \forall i, j$. To preserve the information about the shortest paths, for each element of $P^{\prime}$ that satisfies $w_{p}^{\operatorname{mv}(i)}\left(i_{j}, i_{l}\right)<$ $\min \left(w_{p}\left(i_{j}, i_{l}\right), w_{p}^{\operatorname{mv}(h \neq i)}\left(i_{j}, i_{l}\right)\right)$ we have

$$
p_{i_{j} i_{l}}^{\prime}= \begin{cases}v_{i}, & p_{i i_{l}}^{\prime}=\infty \\ p_{i i_{l}}^{\prime}, & p_{i i_{l}}^{\prime} \neq \infty\end{cases}
$$

Note: if vertex $v_{i}^{p}$, which is to be removed, is adjacent only to one vertex of $G_{p}$, so, as there are no shortest paths passing through $v_{i}^{p}$, the vertex and the incidental edge are simply removed without the shortest path preservation.

We use three parameters for the disassembly stage. $d_{\max }$ " - is the maximum degree of the vertices to be removed. $n_{\min }$ "- is the order of graph $G_{r}$, which is the last (smallest) graph of the shrinking sequence. $I_{\max }$ "- - is the limit of the increasing number of edges after the removal of one vertex. The assignment of values to $d_{\max }, n_{\min }$ and $I_{\max }$ is a problem in itself, which will be discussed elsewhere. The results, which are shown in part 4 , have been obtained by assignment $d_{\max }=$ $I_{\max }=\infty, n_{\min }=1$.

Let us try to remove vertex $v_{i}^{p}$ with all of its $k$ incidental edges and preserve the shortest paths. Denote by $I\left(v_{i}^{p}\right)$ the change in the number of graph edges when the vertex is removed. The removal of $v_{i}^{p}$ itself will decrease the number of edges by $k$, therefore we get $I\left(v_{i}^{p}\right)=-k$. Using the shortest paths preservation and (1), we have: 


$$
I\left(v_{i}^{p}\right)=\left\{\begin{array}{l}
I\left(v_{i}^{p}\right)+1 \\
\text { if } w_{p}\left(i_{j}, i_{l}\right)=\infty \\
\wedge w_{p}^{\operatorname{mv}(i)}\left(i_{j}, i_{l}\right)<w_{p}^{\operatorname{mv}(h \neq i)}\left(i_{j}, i_{l}\right) \\
I\left(v_{i}^{p}\right), \text { else }
\end{array}\right.
$$

Thus we'll obtain the change in the size of graph $G_{p+1}$ relative to $G_{p}$ after the removal of vertex $v_{i}^{p}$. If $I\left(v_{i}^{p}\right)>$ 0 , then the graph size increases, otherwise the graph size decreases or remains the same. Using (3) we expect that the increase of the graph size is bounded above by $I_{\max }$ when a vertex is removed. It follows that vertex $v_{i}^{p}$ can be removed only if $I\left(v_{i}^{p}\right) \leq I_{\max }$.

The selection of the vertices that we are going to remove is performed in the following way. Since vertices meeting $d\left(v_{i}^{p}\right)<3$ can be removed anyway, it follows that vertices should be removed in ascending order of their degrees from 1 to $d_{\max }$. This speeds up the algorithm due to a smaller number of processed vertices with degrees close to $d_{\max }$. After we remove $v_{i}^{p}$, the degrees of the adjacent vertices can change, hence, if we remove $v_{i}^{p}$, the vertices adjacent to $v_{i}^{p}$ should be processed through recursion. The graph disassembly algorithm and an auxiliary algorithm of vertices inspection and removal are on fig. 1 and 2 .

\section{Vertices inspection and removal}

Input: vertex $v_{i}^{p}$, number of vertices $n_{c}, I_{\max }, d_{\max }$ $n_{\text {min }}, p, P^{\prime}$.

Step 1. Vertices inspection

If $d\left(v_{i}^{p}\right)<3$, go to step 2 .

Else $I\left(v_{i}^{p}\right)=-d\left(v_{i}^{p}\right)$. Inspect all pair of vertices $A_{i}^{p}$ and change $I\left(v_{i}^{p}\right)$ by $(3)$.

If $I\left(v_{i}^{p}\right) \leq I_{\max }$, go to step 2 .

Else end of algorithm.

Step 2. Vertex removal

Form a new graph $G_{p+1}=R_{p}\left(v_{i}^{p}\right)$.

Count the weights of the edges between

vertices $A_{i}^{p}$ by (1).

Change the elements of the matrix $P^{\prime}$ by $(2)$.

$n_{c}=n_{c}-1, t=p, p=p+1$.

If $n_{c}=n_{m i n}$, end of algorithm.

Else, while $n_{c}>n_{\min }$ for vertices $v_{i_{l}}^{p}: d\left(v_{i_{l}}^{p}\right)<$ $d\left(v_{i_{l}}^{t}\right)$ do Vertices inspection and removal $\left(v_{i_{l}}^{p}, n_{c}, I_{\max }, d_{\min }, n_{\min }, p, P^{\prime}\right)$.

Fig. 1: Auxiliary algorithm of vertices inspection and removal.

\section{Algorithm of the graph disassembly}

Input: graph $G_{0}=\left(V_{0}, E_{0}, w_{0}\right):\left|V_{0}\right|=n, d_{\max }$,

$$
n_{\min }, I_{\max }, P^{\prime}=\left(p_{i j}^{\prime}\right)_{i=1, j=1}^{n, n}: p_{i j}^{\prime}=\infty, \forall i, j .
$$

Step 0. Data preparation

$d_{c}=1, i=0, n_{c}=n, p=0$.

Step 1. Vertex selection

If $n_{c}=n_{\text {min }}$, end of algorithm.

Else. If $\exists v_{j}^{p} \in V_{p}: j>i \wedge d\left(v_{j}^{p}\right)=d_{c}$, then $i=j$, go to step 2 .

Else. If $d_{c}<d_{\max }$, then $d_{c}=d_{c}+1, i=0$, go to step 1.

Else end of algorithm.

Step 2. Vertices inspection and removal

Vertices inspection and removal

$\left(v_{i}^{p}, n_{c}, I_{\max }, d_{\max }, n_{\min }, p, P^{\prime}\right)$, go to step 1.

Output: graph $G_{r}=G_{p}$.

Fig. 2: Algorithm of the graph disassembly.

\subsection{Microsolution}

Here the APSP for $G_{r}$ is solved. The result of the stage is the distance matrix $M_{r}$ of $G_{r}$. We use matrix $M_{r}^{\prime}=M_{r}$ and recalculate $P^{\prime}$ by

$$
\begin{gathered}
p_{i j}^{\prime}= \begin{cases}p_{i j}^{r}, & p_{i j}^{\prime}=\infty \wedge p_{p_{i j}^{r} j}^{\prime}=\infty \\
p_{p_{i j}^{r} j}^{\prime}, & p_{i j}^{\prime}=\infty \wedge p_{p_{i j}^{r} j}^{\prime} \neq \infty\end{cases} \\
p_{i j}^{\prime}= \begin{cases}p_{i j}^{r}, & w_{r}(i, j)>m_{i j}^{r} \wedge p_{p_{i j}^{r} j}^{\prime}=\infty \\
p_{p_{i j}^{r} j}^{\prime}, & w_{r}(i, j)>m_{i j}^{r} \wedge p_{p_{i j}^{r} j}^{\prime} \neq \infty\end{cases}
\end{gathered}
$$

here $p_{i j}^{r}$ are the elements of the matrix $P_{r}=\left(p_{i j}^{r}\right)$, which corresponds to $G_{r}$. The calculated paths are the shortest ones due to the usage of the distances preservation method. In other words, we have $m_{i j}^{\prime r}=m_{i j}^{r}=$ $m_{i j}^{0}, \forall i, j: v_{i}^{r}, v_{j}^{r} \in V_{r}$.

Obviously, if $G_{r}$ has only one vertex then this stage is skipped and the assembly of the graph starts.

\subsection{Assembly}

Before this stage starts, the graph assembly sequence $S=\left\{G_{0}, G_{1}, \ldots, G_{r}\right\}$ is defined. Here $G_{0}$ "-is the initial graph, $G_{r}$ "-is the smallest graph. The shortest paths between all vertices of $G_{r}$ were found in the previous stage. At the assembly stage we restore the removed vertices in reverse order to their removal. That is we move from $G_{r}$ to $G_{0}$ through $G_{r-1}, G_{r-2}, \ldots, G_{1}$, recalculating the shortest paths for vertex $v_{i}^{r-p}: v_{i}^{r-p+1} \notin$ $V_{r-p+1} \wedge v_{i}^{r-p} \in V_{r-p}$ in each step $p$.

Suppose vertex $v_{i}^{r-1}$ is to be restored, i.e. we move from $G_{r}$ to $G_{r-1}$. Vertex $v_{i}^{r-1}$ is connected with vertices $\left\{v_{i_{z}}^{r-1}\right\}_{z=1}^{k}$ by $k$ edges. Matrix $M_{r}^{\prime}=M_{r}$ of $G_{r}$ was found in the previous step, therefore to find the matrix $M_{r-1}^{\prime}$ of $G_{r-1}$, we only need to calculate the shortest paths from vertex $v_{i}^{r-1}$ to all other vertices of $G_{r-1}$. Other elements of $M_{r-1}^{\prime}$ are assigned equally to the corresponding elements of $M_{r}^{\prime}$, that is $m_{j l}^{\prime r-1}=m_{j l}^{\prime}, \forall j, l: v_{j}^{r}, v_{l}^{r} \in V_{r}$. 
Table 1. Characteristics of graphs used for testing

\begin{tabular}{|c|c|c|c|c|c|}
\hline $\begin{array}{l}\text { Group } \\
\text { graphs }\end{array}$ & $\begin{array}{c}\text { Avg. quantity of } \\
\text { Vertices }\end{array}$ & $\begin{array}{l}\text { Avg. quantity of } \\
\text { Edges }\end{array}$ & $\begin{array}{c}\text { Average } \\
\text { vertex degree }\end{array}$ & $\begin{array}{c}\text { Max } \\
\text { vertex degree }\end{array}$ & Graphs \\
\hline$G 1$ & $10^{3}$ & $2,5 \cdot 10^{3}$ & 2,48 & 6 & \multirow{10}{*}{10} \\
\hline$G 2$ & $2 \cdot 10^{3}$ & $5,21 \cdot 10^{3}$ & 2,6 & 5 & \\
\hline G3 & $3 \cdot 10^{3}$ & $7,88 \cdot 10^{3}$ & 2,62 & 6 & \\
\hline$G 4$ & $4 \cdot 10^{3}$ & $1,07 \cdot 10^{4}$ & 2,68 & 6 & \\
\hline G5 & $5 \cdot 10^{3}$ & $1,33 \cdot 10^{4}$ & 2,66 & 6 & \\
\hline$G 6$ & $6 \cdot 10^{3}$ & $1,58 \cdot 10^{4}$ & 2,63 & 7 & \\
\hline$G 7$ & $7 \cdot 10^{3}$ & $1,85 \cdot 10^{4}$ & 2,64 & 6 & \\
\hline$G 8$ & $8 \cdot 10^{3}$ & $2,08 \cdot 10^{4}$ & 2,6 & 6 & \\
\hline$G 9$ & $9 \cdot 10^{3}$ & $2,36 \cdot 10^{4}$ & 2,62 & 7 & \\
\hline$G 10$ & $10^{4}$ & $2,72 \cdot 10^{4}$ & 2,72 & 7 & \\
\hline$G R$ & $2,1 \cdot 10^{3}$ & $6 \cdot 10^{3}$ & 2,86 & 14 & 20 \\
\hline
\end{tabular}

Table 2. Test results

\begin{tabular}{|c|c|c|c|c|c|c|}
\hline $\begin{array}{c}\text { Group } \\
\text { of graphs }\end{array}$ & $\begin{array}{c}\text { PA avg. } \\
\text { runtime, } \mathrm{s}\end{array}$ & $\begin{array}{c}\text { DB avg. } \\
\text { runtime, } \mathrm{s}\end{array}$ & $\begin{array}{c}\text { PA max } \\
\text { runtime, } \mathrm{s}\end{array}$ & $\begin{array}{c}\text { DB max } \\
\text { runtime, s }\end{array}$ & $\begin{array}{c}\text { Avg. speedup } \\
\text { PA/DB }\end{array}$ & $\begin{array}{c}\text { PA max } \\
\text { deg. of rem. } \\
\text { vert. }\end{array}$ \\
\hline$G 1$ & 0,03 & 1,5 & 0,05 & 1,7 & 50 & 11 \\
\hline$G 2$ & 0,13 & 6,7 & 0,14 & 7,1 & 52 & 12 \\
\hline$G 3$ & 0,31 & 16 & 0,33 & 17 & 52 & 12 \\
\hline$G 4$ & 0,67 & 30 & 0,88 & 32 & 45 & 22 \\
\hline$G 5$ & 1,1 & 48 & 1,2 & 52 & 44 & 16 \\
\hline$G 6$ & 1,5 & 72 & 1,8 & 82 & 48 & 20 \\
\hline$G 7$ & 2,1 & 97 & 2,2 & 104 & 46 & 17 \\
\hline$G 8$ & 2,6 & 131 & 2,8 & 145 & 50 & 21 \\
\hline$G 9$ & 3,7 & 177 & 4,7 & 189 & 48 & 24 \\
\hline$G 10$ & 5,4 & 218 & 6,4 & 230 & 40 & 23 \\
\hline$G R$ & 0,17 & 7,5 & 0,4 & 18,8 & 45 & 17 \\
\hline
\end{tabular}

Since the shortest path from any vertex of $G_{r-1}$ to $v_{i}^{r-1}$ goes through $\left\{v_{i_{z}}^{r-1}\right\}_{z=1}^{k}$, it follows that the distance from $v_{i}^{r-1}$ to any vertex $v_{l}^{r-1}$ of $G_{r-1}$ can be calculated by $m^{\prime r-1}(i, l)=$ $\min _{z=\overline{1, k}}\left(w_{r-1}\left(i, i_{z}\right)+m^{\prime r}\left(i_{z}, l\right)\right)$.

If we move from $G_{r-p+1}$ to $G_{r-p}$ by adding vertex $v_{i}^{r-p}$, to obtaining the distance of matrix $M_{r-p}^{\prime}$, we should use the following

$$
m_{j l}^{\prime r-p}=m_{j l}^{\prime r-p+1}, \forall j, l: v_{j}^{r-p+1}, v_{l}^{r-p+1} \in V_{r-p+1}
$$

$$
\begin{gathered}
m_{i l}^{\prime r-p}=m_{l i}^{{ }^{r}-p}= \\
\min _{z=\overline{1, k}}\left(w_{r-p}\left(i, i_{z}\right)+m^{\prime r-p+1}\left(i_{z}, l\right)\right), \\
\forall l: v_{l}^{r-p+1} \in V_{r-p+1}
\end{gathered}
$$

Denote by $x(l)$ the number $i_{z}$ such that $x(l)$ : $w_{r-p}(i, x(l))+m^{r-p+1}(x(l), l)=$ $\min _{z=\overline{1, k}}\left(w_{r-p}\left(i, i_{z}\right) m^{\prime r-p+1}\left(i_{z}, l\right)\right)$. If any vertex satisfies $w_{r-p}(i, l)>m_{i l}^{\prime r-p} \vee w_{r-p}(l, i)>m_{l i}^{r-p}$ or $p_{i l}^{\prime}=\infty \vee p_{l i}^{\prime}=\infty$, then the respective elements of matrix $P^{\prime}$ should be changed by

$$
\begin{array}{r}
p_{i l}^{\prime}= \begin{cases}v_{i}, & p_{x(l) l}^{\prime}=\infty \\
p_{x(l) l}^{\prime}, & p_{x(l) l}^{\prime} \neq \infty\end{cases} \\
p_{l i}^{\prime}= \begin{cases}v_{l}, & p_{x(l) i}^{\prime}=\infty \\
p_{x(l) i}^{\prime}, & p_{x(l) i}^{\prime} \neq \infty\end{cases}
\end{array}
$$

The assembly algorithm is shown in Figure 3.

\section{Assembly algorithm}

Input: $S=\left\{G_{0}, G_{1}, \ldots, G_{r}\right\}, M_{r}^{\prime}, P^{\prime}, p=1$.

Step 1. Go to a larger graph

If $p \leq r$, count matrix $M_{r-p}^{\prime}$ by $(6),(7)$ and

matrix $P^{\prime}$ by (8) and (9), $p=p+1$.

Else end of algorithm.

Output: matrix $M_{0}^{\prime}$, matrix $P^{\prime}$.

Fig. 3: Assembly algorithm.

\section{Results}

All the tests have been performed on a computer equipped with an Intel Core 2 Duo E8400 (3 GHz) CPU and 2 GBs of RAM on the 32-bit edition of Windows XP. The source code has been written on $\mathrm{C}++$ programming language in Borland $\mathrm{C}++$ Builder 6. Weighted graphs of the USA road networks from an open public source $(G 1-G 10)$ [8] have been used as the test data. Connected subgraphs with sizes from $10^{3}$ to $10^{4}$ of 10 pieces for each size have been derived from graphs $G 1-G 10$. Another set of test data are the graphs of Russian cities' road networks ( $G R$, for detailed specifications look at $[2])$. The details of the test graphs are shown in Table 1 .

Test parameters are $d_{\max }=I_{\max }=\infty, n_{\min }=1$. This means that all the graph vertices except one were deleted. That's why the microsolution stage was not performed. The proposed algorithm's (denote as PA) has been compared to the binary heap implementation of the Dijkstra's algorithm (denote as $D B,[1]$ ), which was performed for every vertex of the test graphs. The test results are shown in Table 2 . 
The proposed algorithm speeds up the solving of APSP an average of 47 times faster in comparison with the Dijkstra algorithm. For each and all test graphs the algorithm is faster than the Dijkstra's algorithm (the minimum speed up is 34 times faster). During the tests, the vertices degrees were increased to a maximum of 17 . This means that the complexity of the vertices removal increases during the disassembly only slightly.

\section{Conclusion}

The proposed algorithm noticeably accelerates the solving of the APSP for graphs of road networks, which is confirmed by the tests. The objects of further research may be the selection of the algorithm parameters based on a fast analysis of graph properties, the modification of the disassembly and assembly order and the scalability issues of the algorithm relative to the increasing of a graphs' dimensions. Also, it is interesting to modify the algorithm to solve the problem quicker, but within a given error.

\section{REFERENCES}

[1] T. H. Cormen, C. E. Leiserson, R. L. Rivest, C. Stein. Introduction to algorithms. 2nd ed., MIT Press, Cambridge, 2001.
[2] A. R. Urakov, T. V. Timeryaev. Using weighted graphs features for fast searching their parameters, Prikl. Diskr. Mat., No.2, 95-99, 2012.

[3] R. Bellman. On a Routing Problem, Quarterly of Applied Mathematics, No.16, 87-90, 1958.

[4] E. W. Dijkstra. A Note on Two Problems in Connexion With Graphs, Numerische Mathematik, No.16, 269-271, 1959.

[5] R. W. Floyd. Algorithm 97: Shortest Path, Communications of the ACM, No.5(6), 345, 1962.

[6] R. Geisberger, P. Sanders, D. Schultes, D. Delling. Contraction Hierarchies: Faster and Simpler Hierarchical Routing in Road Networks, International Workshop on Experimental Algorithms (WEA 2008), 319-333, 2008.

[7] D. B. Johnson. Efficient algorithms for shortest paths in sparse graph, Journal of the ACM, Vol.24, No.1, 1-13, 1977.

[8] 9th DIMACS Implementation Challenge, "-Shortest Paths, Online available from http://www.dis.uniroma1.it/challenge9/download.shtml 